OPEN ACCESS

Edited by:

Christoph Luthi,

Swiss Federal Institute of Aquatic Science and Technology, Switzerland

Reviewed by:

Antoinette Kome,

SNV Netherlands, Netherlands

Isaac Dennis Amoah,

Durban University of Technology,

South Africa

*Correspondence:

Martin Gambrill

mgambrill@worldbank.org

Specialty section:

This article was submitted to Water and Wastewater Management,

a section of the journal

Frontiers in Environmental Science

Received: 24 August 2019 Accepted: 13 December 2019

Published: 11 February 2020

Citation:

Gambrill M, Gilsdorf RJ and Kotwal N (2020) Citywide Inclusive

Sanitation-Business as Unusual: Shifting the Paradigm by Shifting

Minds. Front. Environ. Sci. 7:201.

doi: 10.3389/fenvs.2019.00201

\section{Citywide Inclusive Sanitation-Business as Unusual: Shifting the Paradigm by Shifting Minds}

\author{
Martin Gambrill*, Rebecca J. Gilsdorf and Nandita Kotwal \\ The World Bank, Washington, DC, United States
}

As the world urbanizes, the challenges of urban sanitation increase, with urban population growth dramatically outpacing gains in sanitation access. Total global costs of inadequate sanitation are estimated at USD 260 billion annually, and reaching the SDG urban sanitation targets will require over USD 45 billion each year. 'Business as usual' in urban sanitation-where conventional sewerage and wastewater treatment are considered as the only solution - will not get us to universal safely managed sanitation. Citywide Inclusive Sanitation (CWIS) looks to shift the urban sanitation paradigm, aiming to ensure everyone has access to safely managed sanitation by promoting a range of solutions - both onsite and sewered, centralized or decentralized - tailored to the realities of the world's burgeoning cities. CWIS means focusing on service provision and its enabling environment, rather than on building infrastructure. This shift in paradigm to CWIS requires a shift in mindsets. Governments and development agencies increasingly recognize that historic approaches to urban sanitation have not always worked and new approaches, such as CWIS, are required. Consulting firms need to think differently, and not simply replicate approaches found in high-income countries. Engineering curricula should include the design and management of non-conventional systems and should explore opportunities for leapfrogging to solutions that take full account of the public health and environmental imperatives of urban sanitation. We should rethink the way sanitation infrastructure is funded and challenge approaches that subsidize sewers but not onsite sanitation, that do not embrace innovation and do not consider running costs. CWIS, or 'business as unusual', requires awareness raising and capacity building, the spreading around the world of successful experiences, and the development and use of tools and other resource materials to help better design and implement sustainable urban sanitation services for all. At the World Bank, we see that shifting mindsets toward CWIS principles can be achieved and that there is a growing appetite globally for embracing such principles. We see an important emerging global movement to engage on CWIS by governments and development partners, which provides an unprecedented opportunity to shift the urban sanitation paradigm in the pursuit of universal safely managed sanitation.

Keywords: citywide inclusive sanitation, sanitation, urban, onsite sanitation, sewerage, sanitation financing, sanitation services, sanitation institutions 


\section{SYNOPSIS}

An estimated 55 percent of the global population lack access to safely managed sanitation, which means that over 4.2 billion people do not have sanitation services that ensure their waste is safely handled and treated as well as being reused/disposed of in a safe manner (WHO/UNICEF, 2017). To date, the predominant approach globally to urban sanitation provision has suffered from focusing too heavily on infrastructure investments, especially those supporting conventional sewers and wastewater treatment plants, and has given comparatively little attention to ensuring sustained service delivery through appropriate policy and incentive frameworks, robust service providers, and sound financial planning. This approach has also resulted in many people being left without adequate sanitation services-these most often being the poorest urban residents. The World Bank, along with other sector partners, are prioritizing a new approach to urban sanitation service provision termed 'Citywide Inclusive Sanitation' (CWIS). Over the past three years, the World Bank has been documenting good practices in urban sanitation service delivery from cities across the globe, sharing these positive experiences with governments from an increasing number of cities and countries around the world, and supporting government and service provider counterparts in the design and implementation of investment projects aligned with CWIS principles. This paper attempts to document the World Bank's rational for making this shift and to share the World Bank's initial experiences in engaging with its counterparts on the CWIS agenda.

\section{THE GLOBAL URBAN SANITATION CRISIS \\ The Scale of the Problem}

The urban sanitation sector has been characterized by persistent failures in providing sustainable services to certain populations, the so called unserved and underserved. The challenge of inadequate urban sanitation is further amplified when we consider the need to meet the sanitation target of the Sustainable Development Goals (SDGs) of achieving "access to adequate and equitable sanitation and hygiene for all...paying special attention to the needs of women and girls and those in vulnerable situations" (United Nations, 2015). As cities grow, services need to expand with them such that the disadvantaged residents living in low-income neighborhoods, including those in the ubiquitous, rapidly growing, informal settlements of cities in low- and middle-income countries, are also provided with services.

Currently, over 55 percent of the world's population live in urban areas (UN DESA, 2019). By 2050, the number of city dwellers is projected to increase from 4.2 to 6.7 billion (UN DESA, 2019), with much of this growth occurring in low and lower-middle income countries. This urban population growth dramatically outpaces gains in access to sanitation. The global Millennium Development Goal (MDG) target for sanitation was missed by almost 700 million people and, as of 2015, 2.4 billion people still lacked access to an improved sanitation facility (WHO/UNICEF, 2015). Today, 17 percent of the world's urban dwellers do not have access to basic sanitation (an improved toilet or latrine) and only 43 percent of urban residents have access to "safely managed sanitation," that is to say where their waste is safely managed across the full sanitation service chain, including containment, conveyance, treatment and reuse/safe disposal (WHO/UNICEF, 2017).

Access is particularly low for poor urban households, as is consistently evidenced in data from the WHO/UNICEF Joint Monitoring Programme. For example, the gap in access between the richest and the poorest wealth quintiles in the countries of Southeast Asia exceeded 50 percentage points in 1995 (WHO/UNICEF, 2015). Although access to improved urban sanitation did increase more rapidly among the poorest in this region between 1995 and 2012, significant gaps remained, with only Thailand having managed to close the urban sanitation gap between the rich and the poor. JMP data shows that progress toward achieving the goal of improved sanitation in low- and middle-income countries has, overall, been far slower for the poorest urban inhabitants. Furthermore, while the gap between the richest and the poorest has been reduced in 52 countries, it has increased in 22 others (WHO/UNICEF, 2015). In six out of 14 countries where urban coverage decreased, the gap between the richest and poorest simultaneously increased (WHO/UNICEF, 2019).

The World Bank's Water Supply, Sanitation and Hygiene (WASH) Poverty Diagnostic Initiative (World Bank, 2017a) further demonstrates that differences in levels of wealth, location and other demographic characteristics are associated with significant disparities in the availability and in the quality of WASH services. The WASH Poverty Diagnostic's analyses in 18 countries across the world confirmed the pervasiveness of the gap in infrastructure availability and service delivery between the urban poor and non-poor and between small and large cities, as well as showing disparities between cities across and within geographic regions of the same country. Evidence shows that service delivery levels also vary within and between cities in the same country. Analysis of the coverage of improved water and sanitation by household-level characteristics in 2006 and 2014 in Ecuador shows that a large portion of the improvements at the national level are driven by urban areas, and in particular in the largest cities of Quito and Guayaquil, while unimproved sanitation remains high in secondary cities (World Bank, 2017b). The WASH Poverty Diagnostic for Ethiopia found that, in addition to household access constraints, many low-income residents are without sanitation services in urban centers where many of them work but where the number of public and communal toilets falls far short of demand (World Bank, 2018). And in Bangladesh, we found that only 13 percent of households in the slums of the five largest metropolises have their own sanitation facilities (World Bank, 2017a), further emphasizing the role of shared sanitation in helping meet the immediate needs of urban residents.

Furthermore, a key element of the SDG sanitation target is that household access is not the only important metric, since the safe management of sanitation along the full service chain also needs to be provided. Globally, of those households with access to safely managed services, an estimated 63 percent are connected to sewers, and an additional 32 percent use 
improved sanitation facilities with onsite systems such as flush or pour-flush toilets connected to septic tanks, or dry or wet pit latrines (including facilities shared with other households). However, this number is distorted by data from high-income countries, such as those in North America and Europe, where 93 percent of the population is connected to sewers, which contrasts sharply, for example, with an average sewerage coverage of less than 20 percent in Sub-Saharan Africa (WHO/UNICEF, 2017). Furthermore, although septic tanks and improved pit latrines are considered "safely managed" sanitation facilities, "on-site storage and treatment systems may be compromised due to poor design, damage or flooding" (WHO/UNICEF, 2019). Similarly, having a sewer connection does not mean that the wastewater is safely conveyed and treated before it is discharged or reused.

\section{The Impacts of Insufficient Access to Safely Managed Sanitation}

This lack of access to safe sanitation services within cities results in significant health, environmental and economic burdens. Limited sanitation in urban areas is a major cause of the transmission of enteric diseases such as cholera and those caused by other pathogens. Given the relatively new nature of the SDGs and their measurement/categorization system, there is currently no strong knowledge base regarding the comparative health impacts of safely managed sanitation as compared to those deriving from access to basic sanitation ${ }^{1}$. However, it is widely recognized that safely managed services are needed to ensure that household health is protected, that the health of other urban households is safeguarded, and that the well-being of the environment is maintained.

Diarrhea is the third leading cause of death globally of children under five years old, and an estimated 55 percent of these deaths is attributable to unsafe sanitation [Institute for Health Metrics and Evaluation (IHME), 2018]. Furthermore, poor sanitation and diarrhea are the second and third leading risk factors for stunting worldwide, with 7.2 and 5.8 million attributable cases, respectively (Danaei et al., 2016). In addition, there is evidence that a lack of sanitation leads to lower school attendance, especially for adolescent girls, who require school sanitation facilities to address their menstrual hygiene management needs. A meta-analysis of 138 studies in India found that a quarter of girls did not attend school during menstruation because of a lack of adequate toilets (Van Eijk et al., 2016). Menstrual hygiene management is important for women of all ages: in Ghana, 11.5 million women, or some 80 percent of all Ghanaian women, have no access to waste disposal facilities that adequately separate menstrual hygiene waste from human contact (World Bank, 2016). As is the case with disparities in access rates, lowincome households are also more adversely affected by the poor quality of sanitation services. In Haiti, children in the bottom household income quintile are 2.4 times more vulnerable to the risk of contracting an enteric disease than those in the top quintile (World Bank, 2017a).

\footnotetext{
1 "Basic Sanitation" is defined as the use of improved sanitation facilities which are not shared with other households (JMP; WHO/UNICEF, 2015).
}

Inadequate access to sanitation infrastructure, and the poor subsequent management of the waste streams along the sanitation service chain, also result in substantial negative environmental impacts. Most human activities that use water produce wastewater. As the overall demand for water grows, the quantity of wastewater produced, and its overall pollution loads, are continuously increasing worldwide. Over 80 percent of the world's wastewater, and over 95 percent in some of the leastdeveloped countries, is released into the environment without treatment (United Nations, 2017). Once discharged into water bodies, wastewater is either diluted, transported downstream or infiltrates into aquifers, where it can affect the quality, and therefore the availability, of freshwater supplies. The ultimate destination of wastewater discharged into rivers and lakes is often our seas and oceans with negative consequences for the marine environment (United Nations, 2017). Similarly, onsite sanitation facilities which do not benefit from appropriate collection, conveyance and treatment of the generated fecal sludge and septage also contribute to the growing pollution burden of both groundwater and surface water bodies, as witnessed, for example, in the backwaters of Kerala in India (World Bank, 2013). Calculating the environmental costs of poor sanitation involves the measurement of these direct and indirect impacts on water bodies and their ecosystems. Additionally, given the increasing water scarcity challenges we see in many areas of the world, which are driven in part by climate change and by population increase, as well as by the associated increase in food consumption, the reuse of treated wastewater is becoming an increasingly attractive and feasible option which can provide positive environmental, economic and other benefits to society.

The negative health and environmental impacts associated with a lack of access to safely managed sanitation result in high economic costs which adversely impact economic growth. The total global costs of inadequate sanitation are estimated at USD 260 billion per year or, on average, some 1.5 percent of a country's gross domestic product (GDP) (Hutton, 2012). In cities around the world it is the norm that, even where piped water networks exist, latrine, septic tank, and sewerage coverage lag far behind. To reach the SDG urban sanitation targets, it is estimated that over USD 45 billion will be needed annually to meet the capital investment costs alone (Hutton and Varughese, 2016).

\section{Obstacles to Scaling Solutions}

The shift from the MDGs to the SDGs means that we have to think beyond just constructing additional sanitation facilities. We also need to ensure that the facilities are consistently used by all household members, that the associated human waste is safely managed along the whole sanitation service chain, and that this is the case in all urban neighborhoods, not just in the wealthiest. Furthermore, this access should be available both within and outside of the home environments. These two fundamental shifts-considering more holistic solutions and ensuring access for all-are impeded by the historic approach to urban sanitation.

In recent decades, in many countries around the world, the focus of urban sanitation programs has far too often been solely on the building of infrastructure. In addition, in many cases, these infrastructure investments are undertaken in a disconnected 
way, such that one investment program may construct a sewer network, for example, another build a treatment plant, and yet another would be responsible for the toilets and the related household installations (if the latter are contemplated at all). Without thinking holistically about the sanitation service chain, none of these investments alone will achieve the intended public health and environmental impacts. This focus on infrastructure has resulted in expensive investments in sewer networks and wastewater treatment plants which, all too frequently, have remained under-utilized as the associated links in the sanitation chain, namely effective sewer connections and the necessary household installations are, in many cases, never actually realized. For example, a World Bank review found that, in one project in Cambodia, only 20 percent of eligible households had connected to the sewer network; under a project in Brazil, only 30 percent of households had connected; and through a project in Uruguay, under 40 percent of targeted households had connected to the sewers resulting in five of the eight treatment plants remaining inoperational (World Bank, forthcoming).

Furthermore, by ignoring household demand and household priorities, sanitation infrastructure has at times been poorly adapted to the local context in which it is implemented, and it has all too frequently been assumed that households will change their behaviors regarding their sanitation practices without the incentives for them to do so being appropriately provided. For example, in cultures accustomed to 'washing' (i.e., using water for anal cleansing), as opposed to 'wiping' (using paper to clean), switching to dry, composting latrines would require significant changes in societal norms and individual behaviors.

It is also increasingly clear that solutions to urban sanitation cannot just focus on the sanitation service chain, and that consideration must also be given to how the sanitation services fit within the broader urban context in which they are placed. Sanitation is closely linked to water supply, as well as to solid waste management, drainage, land use and housing development-and this is especially the case in dense urban areas. Most cities around the world undertake sanitation planning and management separately from these other sectors. The resultant development 'silos' consequently hamper progress in the sector. These silos can inhibit the spread of good experiences between cities and between countries, can mean that efficient sanitation service provision does not capitalize upon the economy of scope provided by a good water supply provider in the city, and can result in broader urban development programs not embracing good practice approaches to sanitation provision. The silos can also mean that sanitation programs do not always take account of the broader issues of urban development with which the sanitation services should be aligned and which they should be leveraging.

In addition, sufficient attention has not been given to the enabling environment of policy, governance, institutions, regulation and funding that underpins the sustainable delivery of services. Too often the incentives that are in place-for policy decision making, for funding, for institutional arrangements, for regulation, among others-do not align with the expressed objectives of the sector (Mumssen et al., 2018). We also find that there are too few robust service providers in the sector and that these tend to concentrate on their water supply responsibilities while considering sanitation - if they do consider it at all-to only concern the construction of conventional sewers and wastewater treatment plants. Few such service providers have tariffs which manage to cover the operation and maintenance costs of those sanitation services that they are able to provide, much less their capital costs. The sanitation infrastructure that is built needs to be commensurate with the financial and human resources of those responsible for running it, and the services provided need to be responsive to the demands of urban residents and tailored to the varying realities in which they live.

In order to meet the aspirational goal of sanitation for all, the sector will also have to shift away from considering only sewered sanitation as a solution. Until recently, the sector's focus on infrastructure had also been a focus on conventional, sewered solutions as developed and implemented in high-income economies over many decades. To reach all households with sustainably and safely managed services in the rapidly growing cities of low- and middle-income countries, we will need to consider more than just conventional sewers-we must have a range of solutions which are tailored to the realities of different cities and different neighborhoods, encompassing onsite onsite sanitation and fecal sludge management, through to simplified sewerage approaches. In many ways, however, the sector is underprepared for supporting governments in implementing such a mix of technical solutions. Many, if not most, decision makers still aspire to the building of centralized conventional sewer systems and wastewater treatment plants, irrespective of the costs or the effectiveness of such solutions. Helping governments understand such trade-offs is challenging, particularly where there is limited data available on, and/or no consideration given to, the true capital and operational costs of different technical solutions.

This shift toward embracing a mix of onsite and reticulated technical solutions is further constrained by a lack of technical expertise in the sector. Most existing engineering education curricula continue to concentrate heavily, if not exclusively, on conventional sewerage and wastewater treatment approaches. Many engineering consultancy firms also focus on conventional sewered solutions, without giving the necessary consideration to the appropriateness of these responses in the context in which they will be built, to their operation and maintenance costs and requirements, nor to their effectiveness of reaching poor neighborhoods and households in low- and middleincome countries.

The challenge of access is further complicated when other factors come into play in what are often informal, unregulated settlements, including the issues of space constraints for installing sanitation systems (where do you install a toilet facility in a singleroomed housing unit?), of topographical and related constraints (how do you ensure septic tanks function in high water table environments?), of system design (how do sewer networks function for households that have limited or intermittent water supply?), and of uncertain land tenure (how do you ensure that rents stay affordable for the poorest households while ensuring that landlords can recover their investment and maintenance costs?). 
Business as usual in urban sanitation, where conventional sewerage networks and wastewater treatment plants-which have been installed at great cost in most high-income economies around the world-are considered as the only solution, will simply not allow us to reach universal and safely managed sanitation for all, and especially not for the informal, unplanned low-income areas of rapidly expanding cities across the globe. Even though we have tried these approaches for decades, there are still billions of people globally without access to safely managed sanitation. It is therefore imperative that we revisit our approach to tackling the world's pressing urban sanitation challenges.

\section{CITYWIDE INCLUSIVE SANITATION AT THE WORLD BANK}

\section{Initiating a Global Movement}

Given all of the challenges that exist in the sector, it is encouraging to note the growing number of positive examples of urban sanitation service provision. There are good examples from around the world of efficient service providers which continually strive to deliver urban sanitation solutions to all of their customers including, for example, eThekwini Water and Sanitation in South Africa, Maynilad and Manila Water in the Philippines, and Companhia de Saneamento Ambiental do Distrito Federal (CAESB) together with other utilities in Brazil. There are also examples of innovation in urban sanitation service delivery, ranging from improved fecal sludge management systems and approaches, through 'condominial' and other simplified sewerage systems, to container-based sanitation entrepreneurs. However, getting such experiences to be replicated and scaled up-both within countries and between countriescontinues to be a significant hurdle.

In 2016, a group of development organizations ${ }^{2}$ convened a series of meetings to discuss these urban sanitation challenges and the need for a change in approach in the sector, which resulted in the release of a Call to Action for Citywide Inclusive Sanitation $^{3}$. Since then, the Citywide Inclusive Sanitation (CWIS) principles have strongly resonated with a number of other development partners and with numerous government and service provider counterparts around the world. The CWIS initiative has grown organically-an increasing number of key development partners have started to align their work programs with the principles of CWIS, as articulated in the Call to Action, thereby influencing their government and service delivery counterparts. CWIS-related research work and project support initiatives are also growing in number and in alignment. As a result, a cumulative shift in mindsets is gradually being achieved. The fact that the CWIS principles are not prescriptive but provide general guidance regarding how to respond to the challenges of urban sanitation in low- and middle-income countries, while

\footnotetext{
${ }^{2}$ The Bill \& Melinda Gates Foundation, WaterAid, University of Leeds, Emory University, Plan International and the World Bank.

${ }^{3}$ http://pubdocs.worldbank.org/en/589771503512867370/Citywide-InclusiveSanitation.pdf
}

breaking away from dogma and fixed positions, helps explain this gradual, broad and growing uptake.

\section{The World Bank's Approach to CWIS}

At the World Bank, we have been sharing these ideas with government counterparts across the globe and operationalizing the core Citywide Inclusive Sanitation principles in a range of countries. We emphasize that CWIS challenges us to ensure that everyone has access to safely managed sanitation by promoting a range of technical solutions-both onsite and sewered, centralized or decentralized-which are tailored to the realities of the world's burgeoning cities and which are flexible and adaptable so that, as cities grow and change, sanitation services adapt with them. In promoting this approach, we encourage governments to focus on service provision rather than on building specific infrastructure, which means considering the financial, institutional, policy, regulatory, social and environmental dimensions of the services. We also strive to work across disciplines within the World Bank and with governments to harmonize sanitation solutions with related urban services such as water supply, drainage and solid waste management. Additionally, we promote the thinking that design decisions and their related financial implications should be driven by the consideration of both capital and operational expenditures, rather than focusing solely on the initial investment costs. Furthermore, the World Bank needs to continue working with counterpart governments on their urban sanitation strategies and investment programs to ensure that full consideration is given to how these will encompass service provision to unserved and underserved populations.

We recognize that there is no single solution for responding to the immense urban sanitation backlog in cities of low- and middle-income countries. Instead we believe that locally relevant and innovative solutions need to be developed, tested and scaled up, and that good experiences from around the world need to be spread and adapted as appropriate to their new contexts. Box 1 shows the priorities that the World Bank is encouraging cities to consider when developing their sanitation plans and investment programs.

\section{Operationalizing CWIS Approaches in World Bank Projects}

As the World Bank continues to work with governments across the globe on the planning and the implementation of urban sanitation interventions that align with the CWIS principles, we encounter a wide range of unique challenges that require specific responses. Each city and, in many cases, each neighborhood, represents a new opportunity to learn further about what CWIS can look like in practice. Nevertheless, as the World Bank works with its government counterparts to implement CWIS approaches, there are some emerging common challenges that we have seen come to light in a number of cities and countries where our urban sanitation portfolio is active.

We see that this shift in paradigm to Citywide Inclusive Sanitation requires a concomitant shift in mindsets. Development agencies, which collectively invest billions of dollars in urban sanitation projects each year, have started to 
BOX 1 | Promoting Citywide Inclusive Sanitation at the World Bank.

\section{A CWIS project is where...}

$\checkmark$ Everybody benefits from adequate sanitation service delivery outcomes that meet user aspirations and that protect the health of users.

$\checkmark$ Human waste is safely managed along the whole sanitation service chain ensuring protection of the environment and of human health.

$\checkmark$ A diversity of appropriate technical solutions is embraced, combining both onsite and sewered solutions, in either centralized or decentralized systems, with consideration of resource recovery and re-use.

$\checkmark$ Cities demonstrate political will, technical and managerial leadership, and identify new and creative long-term funding options for sanitation.

$\checkmark$ Institutional arrangements and regulations, with well-aligned incentives, are in place for the operation and maintenance of the full sanitation service chain.

$\checkmark$ Funding is allocated for non-infrastructure aspects of service delivery, such as capacity building, household engagement and outreach, and sanitation marketing.

$\checkmark$ Complementary urban services, including water supply, drainage, graywater management and solid waste management, are incorporated into sanitation planning.

$\checkmark$ Activities are included to target specific unserved and underserved groups, such as women, ethnic minorities, the urban poor and people with disabilities.

recognize that the historic approach to urban sanitation has not always worked and that new approaches, such as CWIS, need to be considered. Similarly, the different levels of government involved in urban sanitation, and the entities responsible for service delivery, are increasingly recognizing the need for such a shift in approach. Equally importantly, we see that the consulting firms who assist governments in the preparation of master plans, engineering designs and related studies also need to think differently and be open to approaches that do not simply replicate those found in high-income countries, where the technical, social and institutional realities are very different. Shifting the mindsets of this range of actors is not easy, but it needs to be tackled if we are to achieve the urban sanitation SDG target. At the World Bank we have consequently been providing technical assistance to our government counterparts through capacity building events, knowledge exchanges and workshops, in which we share good practice approaches to urban sanitation service provision, and where we discuss how such experiences can be transferred, adapted and scaled up to new settings.

We also see that the existing cadre of managers, engineers, technicians and others working in the sector are all learning together about new approaches to responding to urban sanitation challenges. The educational systems in which sector professionals are taught, however, tend to focus on the so-called conventional solutions that have been developed over many decades in high-income countries but that cannot be simply imported into the contexts of rapidly expanding cities in low- and middle-income countries. We nevertheless continue to witness consulting firms still only wanting to focus on conventional sewer networks and wastewater treatment: additional time for advocacy and learning is needed so that they, too, start to embrace the planning and implementation of projects that include the necessary mix of technologies and services which are commensurate with the realities where they will be applied. We need to teach the engineers of tomorrow that, although seemingly obvious, we cannot design sewer systems in cities where there is insufficient water supply, just as we cannot design septic tank systems in situations where the ground conditions offer no effluent infiltration capacity. In addition, the implementation of CWIS requires a number of non-engineering skillsets including, for example, business skills for understanding the dynamics of formal and informal pit and tank emptiers, private sector oversight skills for services that are contracted out by public utilities, an understanding of the role of behavior change to facilitate the uptake of new approaches to sanitation service delivery, and household engagement skills for all service providers. In an effort to address these shortfalls, the World Bank has been undertaking capacity building and training events, as well as developing tools and guidance documents, for government counterparts and others in the sector, including doing so in collaboration with academia through online and in-person capacity building initiatives. These activities address a wide range of technical solutions as well as a suite of the non-technical dimensions of urban sanitation service delivery.

We see that those who are responsible for designing and implementing interventions, still struggle with how to operationalize fecal sludge management (FSM) systems and reuse at a citywide scale, including confronting difficulties related to: (i) the design and procurement of FSM service delivery arrangements which encompass the logistics of collection and conveyance, the siting of transfer stations and treatment plants, and the regularization of the informal private sector emptiers; and (ii) the review of roles and responsibilities with respect to the funding, the contracting out, the delivery and the regulation of FSM services. We also encounter difficulty in advancing the reuse agenda for both wastewater effluent and for fecal and wastewater sludge against prevailing regulatory standards and in the context of a general lack of understanding of business models and market demand for reuse products. Furthermore, many sewerage projects fail to get a critical mass of households to connect to the networks, thus resulting in wastewater treatment plants not operating at their design capacities and, consequently, the associated public health and environmental benefits not being accrued. In order to fill these knowledge gaps for operationalizing CWIS, the World Bank has been developing specific operational tools (generic terms of reference, guidance notes, technical manuals, planning and costing tools, etc.) designed to assist World Bank project teams and their government counterparts in better designing and implementing urban sanitation projects. These materials are complemented with a range of curated resources designed to support our government counterparts in considering and designing alternative approaches.

We also see that we need to rethink the way we fund sanitation infrastructure and we should challenge those who deem, for example, that onsite sanitation services, which overwhelming target the poor, should not be subsidized when sewer systems and wastewater treatment plants, which tend to benefit the 
richer, planned areas of cities, have consistently benefited from subsidies. We need to carefully consider the capital and the running costs and complexities of the systems we design and ensure that they are commensurate with the capacities of those responsible for operating them.

In many situations, the modalities of sanitation service provision also need to be reconsidered. We should harness, where appropriate, the benefits of the economies of scope that can be achieved by robust service providers delivering both water supply and sanitation services together, thereby leveraging the financial, organizational, management and human resources capacity, and the billing systems, that such combined service provision offers. Service provider arrangements and institutional and regulatory set-ups in which the traditional water supply utility only sees, or is only permitted to work with, conventional sewers, need rethinking. We should encourage service providers to embrace a culture of research and development aimed at improving service provision and customer satisfaction while lowering costs. In addition to such context-specific research and development, we also need to further develop an aligned research agenda at the global level. We are working with government counterparts on the development of sanitation business and management models, be they public, private or a mix of both, that are able to reach customers with services.

We also need to embed an inclusive approach to sanitation service provision and to design and manage sanitation systems based on the context in which they will be placed. The World Bank has conducted research on women in 28 water utilities (World Bank, 2019c) exploring the barriers women experience when entering into, remaining in, and advancing within the water and sanitation sector, looking specifically at the issue of the working conditions of women in water and sanitation utilities. Furthermore, the World Bank is looking at existing research on female-friendly public and communal toilets [United Nations Children's Fund (UNICEF), WaterAid and Water and Sanitation for the Urban Poor (WSUP), 2018], in order to better incorporate these design considerations into our sanitation projects. There is also an emerging set of projects where the World Bank has been collaborating with governments to formalize the work of septage/fecal sludge emptiers and to advance their health and safety operating environments. Projects that we are funding in Kinshasa and Lusaka, for example, are supporting the formalization of pit/septic tank emptiers, while also improving their working conditions. The World Bank also includes menstrual hygiene management initiatives under a growing number of the projects it funds, with a view to making the associated interventions more accessible to women and girls.

Encouragingly, there are a number of sanitation service innovations that can be drawn upon when designing investment programs, including: new innovations in treatment and reuse technologies; non-conventional approaches to implementing sewers; container-based sanitation (CBS) service delivery models (World Bank, 2019a); source separation of blackwater and graywater; and the use of mobile technology for improving billing and for the monitoring and evaluation of service provision. However, many of these innovations have failed to reach scale given a generalized conservatism within the urban sanitation
BOX 2 | Current World Bank CWIS engagements.

\begin{tabular}{l|l|l}
\hline - Angola & • Egypt & - Liberia \\
- Bangladesh & - Ethiopia & - Malawi \\
- Benin & - eSwatini & - Mozambique \\
- Bolivia & - Ghana & - Nigeria \\
- Botswana & - Haiti & - Rwanda \\
- Burundi & - India & - Solomon Islands \\
- Cambodia & - Kenya & - Tri Lanka \\
- Colombia & - Lanania \\
- Côte d'Ivoire & - Uganda \\
- Democratic Republic of Congo & - Lesotho & - Uruguay \\
- Dominican Republic & & - Zambia \\
& &
\end{tabular}

sector, limited financing focused on innovation, the time it takes to pilot and refine new approaches, and a general lack of familiarity with many of these emerging practices. Financial support to aid start-ups in moving from pilot to larger scale could, through partnerships with more established or bigger service providers and other strategic entities, enable the more rapid implementation and scale up of such innovation.

Achieving the additional take up and roll out of Citywide Inclusive Sanitation will require the further development of relevant guidance and reference material and will involve the continued spreading of good practice approaches from one side of the world to the other. Such business as unusual that CWIS represents will entail significant efforts to raise awareness and build capacity-not only for those entering engineering school but also for those in government and those managing/working for service providers, both in the public and private sectorswho influence decisions today. We have seen at the World Bank how the shifting of mindsets to embrace CWIS principles can be achieved in relatively short time periods when tailored awareness raising and capacity building programs are carried out at the right time and with the appropriate content within the investment project cycle.

\section{Examples of CWIS in World Bank Projects}

At the World Bank we find that there is appetite for moving toward the principles of CWIS in our existing and new urban sanitation investment projects, in low- and middle-income countries alike and, increasingly, we see government and service provider counterparts interested in adopting CWIS approaches. We currently have engagements in a diversity of countries around the world (see Box 2) in which we are advancing CWIS principles, at large and small scales, be it in single or multiple cities in a given country. The CWIS team at the World Bank provides expertise to support World Bank and government project teams in operationalizing and embedding CWIS principles within their urban sanitation investments.

\section{CWIS Planning}

In line with the CWIS principles, urban sanitation planning processes are also in need of revisiting. The World Bank has been working with governments in the undertaking of 
participatory planning that focuses on the types of services that will be delivered, on the institutions needed to support these services, and on the financing streams for both their capital and operational expenses. In lieu of focusing exclusively on sewerage master plans, these planning processes begin with a clear assessment of existing sanitation services-for the full service chain and across all parts of the city. The methodology then aims to define the objectives of the sanitation planning process and to produce a plan which states the types of technologies and services that will be used in each neighborhood/division of the city, with the specific units/levels of planning being defined by the city decision makers and other key stakeholders.

The scale at which such strategic sanitation planning occurs may also vary depending on local realities and needs. For instance, in Kenya the World Bank has supported the government in undertaking an approach that provides a planning process at the county level termed Countywide Inclusive Sanitation, which encompasses the planning of sanitation services along the rural/small town/peri-urban/urban continuum.

\section{CWIS Project Implementation}

In Ethiopia, the World Bank is funding a large investment project that focuses on expanding access to urban sanitation services for 2.5 million people, while strengthening the institutions that provide these services. The project, which includes activities in the capital and in 22 other cities across the country, will support sewered and onsite solutions and incorporate consideration of the full sanitation service chain. The project is working with government counterparts and consulting firms to improve the planning process-both in terms of rapid assessments and the identification of priority 'no regret' investments. An issue of particular importance is the building of capacity of the country's water service providers whose mandate has recently been expanded to include urban sanitation services. One way of sharing knowledge is through experience exchanges between cities within the country. The World Bank's Citywide Inclusive Sanitation Costing and Planning Tool (World Bank, 2019b) has also been used under the project to help decision makers think through the technical feasibility and financial implications of different approaches to sanitation service delivery along the whole service chain.

In Bolivia, the World Bank has been providing technical assistance support to different levels of government and to service providers to help advance the country's urban sanitation agenda, with a focus on participatory CWIS strategic planning, on better assessing and improving fecal sludge management, on piloting the use of non-conventional sewers, and on improving the technical, financial, regulatory and customer outreach approach to connecting unconnected households to existing conventional sewer networks.

In Bangladesh, the World Bank is working with service providers and with city and municipal governments to advance urban sanitation provision for all by embracing approaches that include the rehabilitation and provision of conventional centralized sewers, but in conjunction with the implementation of approaches and policies to ensure that households actually connect to them, and the implementation of simplified sewer systems and of onsite sanitation and fecal sludge management, in both centralized and decentralized contexts.

For the Greater Accra Metropolitan Area in Ghana, the World Bank is supporting the government in implementing multiple technological and financing innovations in its efforts to provide sanitation services for all. The project has funded the implementation of a mobile money platform for households to save toward the cost of a toilet, representing a new, innovative use of mobile savings schemes. The savings platform allows participation by those who cannot otherwise access the traditional banking sector and who are often the poorest and thus also the most likely to lack proper sanitation at home. The project has also supported an innovation challenge fund that resulted in new technologies (such as different types of household level sanitation containment, and treatment solutions for high flood prone areas) becoming available on the market that are now being provided for low income households under the project.

\section{CWIS Knowledge Sharing}

The World Bank's global CWIS initiative is designed to bring about a shift in the mindsets of different groups of stakeholders through the implementation of tailored approaches to knowledge sharing. This includes providing awareness raising and capacity building activities for World Bank staff and their government counterparts (at the national, city and service delivery levels) who are responsible for the preparation and implementation of Bank-funded urban sanitation investment programs. A key aspect of this work involves the undertaking of knowledge sharing and capacity building activities, either tailored to a specific country or project, or designed for a group of countries and investment programs. A central element of these activities is the promotion of peer-to-peer learning from global good practice urban sanitation service delivery, and the sharing of tools and other resource materials to improve project design and implementation. The World Bank has delivered a number of country and regional CWIS knowledge and learning events in recent years that have brought together stakeholders from a broad range of locations ${ }^{4}$.

Feedback from these national and regional knowledge and learning events has consistently shown very high ratings of satisfaction regarding their content and design, while highlighting the importance of the following elements: (i) presenting compelling examples of urban sanitation service delivery for all; (ii) sharing resource materials and tools developed to help better design and implement such interventions; (iii) providing a balance between presenting new knowledge and demonstrating examples of implementation in practice; (iv) creating opportunities to maximize the exchange of experiences within and between countries; (v) developing

\footnotetext{
${ }^{4}$ Regional CWIS Knowledge \& Learning events have so far been conducted in: Durban, South Africa (December 2016); Brasilia, Brazil (March 2018); Accra, Ghana (May 2018); and Kampala, Uganda (November 2019), with the participation of government counterparts and Bank staff as well as experts from around the world. Country-focused events have been conducted in Bangladesh, Ethiopia, India, Indonesia and Kenya, among others.
} 
action plans to help concretize learning from the events; and (vi) providing suggestions on how to maintain momentum in advancing CWIS principles and practices as follow up to the events. In order to sustain the knowledge sharing that occurs at these events, the World Bank's CWIS team and regional focal points support ongoing dialogue with government counterparts.

\section{Partnerships for CWIS}

As described above, a significant amount of the World Bank's efforts in CWIS is directed toward working with counterparts from different levels of government and from service providers to advance the concepts and the practice of sustainable urban sanitation service provision through the adoption and adaptation of CWIS principles. These partnerships between governments and the World Bank are at the heart of our work and of our modus operandi during both project preparation and implementation. In addition, we are also working across the world with a number of other key partners in the sector to advance the concepts of Citywide Inclusive Sanitation, including with other multilateral development banks, bilateral donors and various other development partners.

We are partnering with the Bill \& Melinda Gates Foundation under a new Urban Sanitation Innovation Partnership to help both organizations and their government counterparts in working toward 'proof of scale' for innovative sanitation delivery services for all, with a focus on sustainable services for the poor. We are also working with a number of bilateral organizations. In Angola, for example, we are co-financing a project with the Agence Française de Développement that will support strategic sanitation planning in nine secondary cities and will pilot sanitation service provision for 35,000 people.

We are collaborating with WaterAid, GIZ and other development partners on the shared, community and public sanitation agenda; with WaterAid, the World Health Organization and the International Labour Organization on the challenges and realities facing sanitation workers; with pS-Eau on non-conventional approaches to designing and implementing sewers; with Eawag-SANDEC and the Gates Foundation on training material for a new generation of urban sanitation sector professionals; and with other nongovernmental organizations, think tanks and research entities on various CWIS-related initiatives.

\section{CONCLUSION}

As the world urbanizes, the challenges of urban sanitation provision increase, with urban population growth dramatically outpacing gains in sanitation access. In low- and middle-income countries, much of this urban growth is taking place in rapidly expanding, unplanned informal settlements, where the provision of sanitation services is especially challenging. The economic, social and development impacts from a lack of sanitation on health, mortality and productivity are compounded by negative impacts on the environment and, ultimately, on economic growth. In cities across the world, it is the norm that, even where piped water networks exist, sanitation coverage lags far behind.
To reach the SDG urban sanitation goal of providing safely managed sanitation for all requires us to think beyond business as usual, in which conventional sewerage networks and wastewater treatment plants are seen as the only solution. Citywide Inclusive Sanitation is designed to shift the urban sanitation paradigm, with the aim of ensuring that everyone has access to safely managed sanitation by promoting a range of technical solutions-both onsite and sewered, centralized or decentralized-which are tailored to the realities of the world's burgeoning cities and which are flexible and adaptable so that, as cities grow and change, the sanitation services adapt with them. CWIS means focusing on service provision rather than on building infrastructure, while considering the financial, institutional, policy, regulatory and social dimensions of the services, and while harmonizing sanitation solutions with related urban services.

This shift in paradigm to Citywide Inclusive Sanitation requires a shift in mindsets. Governments and their development partners are increasingly recognizing that historic approaches to urban sanitation have not always worked and that new approaches, such as CWIS, are required. Consulting firms need to think differently, and not simply replicate approaches found in high-income countries. Engineering curricula should include the design and management of non-conventional systems and should explore opportunities for leapfrogging to solutions that take full account of the public health and environmental imperatives of urban sanitation.

CWIS, requires awareness raising and capacity building and the spreading of successful practices from one side of the world to the other. At the World Bank, we find that there is appetite for moving toward CWIS in our urban sanitation investment projects in low- and middle-income countries alike and, increasingly, we see government and service provider counterparts interested in embracing such approaches. We also see an important emerging global movement to engage on CWIS, which provides the sector with an unprecedented opportunity to shift the urban sanitation paradigm in the pursuit of universal safely managed sanitation. However, such paradigm shifts are by no means easily embraced, and efforts must continue in advocacy, awareness raising and capacity building in order to shift entrenched thinking. We have seen that shifting mindsets to move the paradigm to approaches which embrace CWIS principles can be achieved in relatively short time periods, if tailored awareness raising and capacity building programs are carried out at the right times and with the appropriate content throughout the investment project cycle.

At the World Bank we have been contributing to bringing about such changes through the curation and creation of knowledge products, including the documentation of good practices, the development of a suite of guidance material and tools, the provision of targeted technical assistance, and the delivery of knowledge sharing events-all aimed at improving the preparation and implementation of urban sanitation investment programs.

Despite these advances, there is a need to further expand our knowledge and experience with regard to the design and implementation of Citywide Inclusive Sanitation projects in 
practice, and to do so at scale. To that end, the World Bank continues to leverage its partnerships in the sector to further document what is working, what is not, and what areas still require further innovation and analysis.

In summary, there is a huge challenge confronting the world if it is to reach the SDG target of providing safely managed urban sanitation for all. If this ambitious goal is to be attained, governments and service providers, together with their

\section{REFERENCES}

Danaei, G., Andrews, K. G., Sudfeld, C. R., Fink, G., McCoy, D. C., Peet, E., et al. (2016). Risk factors for childhood stunting in 137 developing countries: a comparative risk assessment analysis at global, regional, and country levels. PLoS Med. 13:e10 02164. doi: 10.1371/journal.pmed.1002164

Hutton, G. (2012). Global Costs and Benefits of Drinking-Water Supply and Sanitation Interventions to Reach the MDG Target and Universal Coverage. Geneva: World Health Organization.

Hutton, G., and Varughese, M. (2016). The Costs of Meeting the 2030 Sustainable Development Goal Targets on Drinking Water, Sanitation, and Hygiene. Technical Paper, Water and Sanitation Program (WSP). Washington, DC: World Bank.

Institute for Health Metrics and Evaluation (IHME) (2018). GBD Compare Data Visualization. Seattle, WA: IHME; University of Washington. Available online at http://vizhub.healthdata.org/gbd-compare (accessed August $15,2019)$.

Mumssen, Y., Gustavo, S., and Kingdom, B. (2018). Aligning Institutions and Incentives for Sustainable Water Supply and Sanitation Services: Report of the Water Supply and Sanitation Global Solutions Group, Water Global Practice, World Bank. Washington, DC: World Bank.

UN DESA (2019) United Nations, Department of Economic and Social Affairs, Population Division. World Urbanization Prospects: The 2018 Revision. (ST/ESA/SER.A/420). New York, NY: United Nations.

United Nations (2015). Transforming our World: The 2030 Agenda for Sustainable Development. Resolution adopted by the General Assembly on 25 September 2015. Available online at: http://www.un.org/ga/search/view_doc.asp?symbol= A/RES/70/1\&Lang=E.

United Nations (2017). UN World Water Development Report: Wastewater, the Untapped Resource. New York, NY: United Nations.

United Nations Children's Fund (UNICEF), WaterAid and Water and Sanitation for the Urban Poor (WSUP) (2018). Female-Friendly Public and Community Toilets: A Guide for Planners and Decision Makers. London: WaterAid. Available online at: washmatters.wateraid.org/female-friendly-toilets

Van Eijk, A. M., Sivakami, M., Thakkar, M. B., Bauman, A., Laserson, K. F., Coates, S., et al. (2016). Menstrual hygiene management among adolescent girls in India: a systematic review and meta-analysis. BMJ 6:e010290. doi: 10.1136/bmjopen-2015010290

WHO/UNICEF (2015). Progress on Sanitation and Drinking Water: 2015 Update and MDG Assessment. Geneva: WHO and UNICEF,. development partners, will need to continue to collectively work toward changing the thinking behind, and the approaches to, urban sanitation service delivery.

\section{AUTHOR CONTRIBUTIONS}

All authors listed have made a substantial, direct and intellectual contribution to the work, and approved it for publication.

WHO/UNICEF (2017). Available online at: https://washdata.org/data

WHO/UNICEF (2019). Progress on Household Drinking Water, Sanitation and Hygiene 2000-2017. Special Focus on Inequalities. New York, NY: United Nations.

World Bank (2013). Septage: Kerala's looming sanitation challenge. World Bank Blogs. Available online at: https://blogs.worldbank.org/water/septage-kerala-slooming-sanitation-challenge.

World Bank (2016). Measuring the Influences of School WASH-Based MHM Interventions on Adolescent Girls' Educational Engagement in Accra, Ghana Infographic. The Greater Accra Metropolitan Area Sanitation and Water Project, World Bank. Available online at: https://menstrualhygieneday.org/wpcontent/uploads/2018/06/WB_infographicMHMGhana.pdf

World Bank (2017a). Reducing Inequalities in Water Supply, Sanitation, and Hygiene in the Era of the Sustainable Development Goals: Synthesis Report of the WASH Poverty Diagnostic Initiative. WASH Synthesis Report. World Bank, Washington, DC.

World Bank (2017b). Pipe(d) Dreams Water Supply, Sanitation, and Hygiene Progress and Remaining Challenges in Ecuador WASH Poverty Diagnostic. World Bank, Washington DC.

World Bank (2018). Maintaining the Momentum while Addressing Service Quality and Equity: A Diagnostic of Water Supply, Sanitation, Hygiene, and Poverty in Ethiopia. WASH Poverty Diagnostic. Washington, DC: World Bank.

World Bank (2019a). Evaluating the Potential of Container-Based Sanitation. World Bank, Washington, DC.

World Bank (2019b). The Citywide Inclusive Sanitation Costing and Planning Tool. Available online at: http://200.58.79.50/fmi/webd/CWIS\%20Planning\%20Tool $\% 201 \_4$

World Bank (2019c). Women in Water Utilities: Breaking Barriers. World Bank, Washington, DC.

World Bank (forthcoming). Connecting the Unconnected. World Bank, Washington, DC.

Conflict of Interest: The authors declare that the research was conducted in the absence of any commercial or financial relationships that could be construed as a potential conflict of interest.

Copyright (c) 2020 Gambrill, Gilsdorf and Kotwal. This is an open-access article distributed under the terms of the Creative Commons Attribution License (CC BY). The use, distribution or reproduction in other forums is permitted, provided the original author(s) and the copyright owner(s) are credited and that the original publication in this journal is cited, in accordance with accepted academic practice. No use, distribution or reproduction is permitted which does not comply with these terms. 